\title{
Research
}

\section{Variation in UV-Mediated Damage Recovery Among Pseudoidium neolycopersici Isolates: Possible Mechanisms}

\author{
Ranjana Pathak | Aruppillai Suthaparan ${ }^{\dagger}$ (D) |
}

Department of Plant Sciences, Faculty of Biosciences, Norwegian University of Life Sciences, 1432 Ås, Norway

\footnotetext{
† Corresponding author: A. Suthaparan; aruppillai. suthaparan@nmbu.no
}

Accepted for publication 13 March 2021.

\section{Author contributions}

Design and execution of experiments by R.P. and A.S., data analysis and manuscript draft by R.P. and manuscript revision by A.S.

\footnotetext{
Funding

This research was financially supported by the Research Council of Norway, and Norwegian University of Life Sciences Ph.D. grant 1207051033

$\boldsymbol{e}$-Xtra: Supplementary materials are available online.
}

\begin{abstract}
The cryptochrome/photolyase family (CPF) consists of a diversified class of flavoproteins that are evolutionarily related. Although their domain architecture is highly conserved, they perform entirely different physiological functions. Previous studies have confirmed the presence of a functional photolyase in Pseudoidium neolycopersici, which repairs UV-C-induced DNA damage by using near UV-A/blue light as an energy source. Similar doses of UV-C treatment followed by incubation with dark or blue light was tested on conidia germination of five isolates of $P$. neolycopersici collected from different regions of Norway and the Netherlands. The results showed variations in the effect of UV on germination and germination recovery under blue light incubation after UV treatment. Evolutionary studies confirmed that $P$. neolycopersici photolyase is highly conserved among different isolates of $P$. neolycopersici and among different species. All CPF members have a core domain consisting of an identical cofactor, flavin adenine dinucleotide (FAD), and an additional photoantenna chromophore. An amino acid sequence analysis demonstrated that CPF members have highly conserved $C$ terminals compared with their $N$ terminals, because FAD binds in the C-terminal region. When compared with other CPF members, varying lengths of $\mathrm{N}$ and $\mathrm{C}$ terminals were noticed in $P$. neolycopersici photolyase and putative cryptochrome, respectively. Further research using comparative genomics targeting pyrimidine base composition, the role of regulatory elements, including promotor architecture, and the characterization of optical properties of native photolyase among isolates may help to explain the differences in the biological responses of conidia germination of $P$. neolycopersici treated with UV.
\end{abstract}

Keywords: photolyase, polymorphism, powdery mildew, tomato, ultraviolet

The author(s) declare no conflict of interest. 
Powdery mildew caused by Pseudoidium neolycopersici is a significant threat to tomato production worldwide (Lebeda et al. 2017). Several strategies, including the application of fungicide and breeding for disease resistance, are currently used in the management of powdery mildews (Jones et al. 2001). However, the rapid emergence of fungicide-resistant pathogen isolates with the potential to quickly overcome the vertical resistance developed in crop varieties, coupled with high research costs, is discouraging investment in the development of fungicides and more resistant varieties. Furthermore, environmental and consumer concerns regarding the use of chemicals in crop production demands alternative strategies which are environmentally conscious. The use of optical radiation (ultraviolet [UV], visible, and infrared) in the management of powdery mildew pathosystems has been reported to be an efficient and environmentally friendly option for a wide range of crops at the experimental greenhouse level (Suthaparan et al. 2012a, b, 2014, 2016a). The efficiency of this strategy is dependent on the selection and combination of effective wavelengths, application time of the day (within a 24-h period), dose (level of irradiance and duration of exposure), and frequency (number of applications within the pathogen development cycle) (Suthaparan et al. 2016a, b, 2017, 2018). Within the optical radiation range tested, a UV range of 250 to $290 \mathrm{~nm}$ and a red-light range of 635 to $680 \mathrm{~nm}$ have shown a potential to combat powdery mildews (Suthaparan et al. 2016b, 2018). UV has been known to induce damage to DNA, primarily in the form of the formation of dimers between two adjacent pyrimidine nucleotides on the same strand (Sinha and Häder 2002). The existence of this damage inhibits the transcription process of genes and replication process of DNA, which then leads to cell cycle arrest and, ultimately, cell death (Jenkins et al. 2000; Sancar 1994; Tornaletti et al. 1999).

Cryptochromes (CRY) and photolyases (PHR) are evolutionarily related flavoproteins with structural similarity; however, they have distinct physiological roles (Kavakli et al. 2017). The key difference in gene structures between CRY and PHR is that CRY have an extra C-terminal extension, with great variation among different groups of CRY (Chaves et al. 2006; Mei and Dvornyk 2015). All CRY/PHR family (CPF) members contain a core domain, which is highly conserved and subjected to carrying out catalytic activity (Chaves et al. 2006). PHR-mediated DNA damage repair is the most efficient mechanism in relation to other reported mechanisms of recombination repair and nucleotide excision repair (Jans et al. 2005; Manova and Gruszka 2015). PHR are the members of the blue light photoreceptors, which absorb photons in the near-UV/blue regions (300 to $500 \mathrm{~nm}$ ) as an energy source and repair damage caused by UV in DNA (Sancar 2008; Thompson and Sancar 2002). The enzyme was first functionally characterized in Escherichia coli, followed by several other organisms, including fungi, with the exception of placental mammals and a few other species (Mei and Dvornyk 2015; Sancar et al. 1984; Yajima et al. 1991).

As obligate biotrophic phytopathogens with hyaline appearance (except the ascocarp), fungi that cause powdery mildews were assumed to be host dependent for environmental signaling, especially in relation to optical environments. Recent studies have confirmed the presence of genes similar to all major classes of photoreceptors in the range of fungal species that cause powdery mildews in grapevine, tomato, cucumber, and strawberry (Pathak et al. 2017; Suthaparan et al. 2012b). An in-depth sequence analysis of $P$. neolycopersici, Podosphaera xanthii, and $P$. aphanis genomes revealed the presence of three putative genes similar to CPF (unpublished data). Further studies on Pseudoidium neolycopersici CPF genes with the assistance of an $E$. coli expression system revealed that only one among the three CPF genes is a functional PHR, with the possible presence of flavin adenine dinucleotide (FAD) as a catalytic cofactor and 5,10-methenyltetrahydrofolate (MTHF) as a photoantenna cofactor. The action spectra of $P$. neolycopersici conidia germination recovery and survival of $E$. coli cells both reveal that the action spectra of $P$. neolycopersici PHR falls within the 360- to 460-nm range (Pathak et al. 2020).

However, preliminary studies have shown variation in the efficiency of nighttime UV treatment in the suppression of powdery mildews in cucumber and tomato (unpublished data). In this study, we tested the $P$. neolycopersici isolates collected from different geographical regions for (i) UV tolerance in the conidial germination process, (ii) nucleotide sequence similarity of CPF genes, (iii) phylogenetic classification, and (iv) amino acid sequence conservation of $\mathrm{CPF}$ genes in relation to other CPF-like genes available in NCBI.

\section{MATERIALS AND METHODS}

\section{Collection and maintenance of $P$. neolycopersici isolates}

Four isolates of $P$. neolycopersici collected from different locations in Norway and one isolate from the Netherlands were used in this study. These isolates were named according to their collection locations: Akershus (As), Vestfold (Vf), Finnøy (Fn), and Sandnes (Sn) in Norway, and the Netherlands (Nl).

Powdery mildew-sensitive Espero tomato plants were used to maintain all five isolates of powdery mildew collected from different regions. Tomato plants were propagated by seed and grown in controlled-environment chambers with an air temperature and relative humidity (RH) of $20 \pm 2^{\circ} \mathrm{C}$ and $75 \pm 5 \%$, respectively. A daily light cycle of $16 \mathrm{~h}$ with an irradiance of $110 \pm 10 \mu \mathrm{mol} \mathrm{m}^{-2} \mathrm{~s}^{-1}$ was provided using high-pressure mercury lamps (Powerstar HQI-BT 400 W/D day light; OSRAM GmbH).

Disease-free Espero tomato leaflets were surface disinfested by soaking in 3\% ( $\mathrm{vol} / \mathrm{vol})$ sodium hypochlorite solution for $3 \mathrm{~min}$, followed by three sequential rinses with sterilized distilled water, and placed in 9-cm Petri dishes containing $1 \%$ agar (wt/vol) and $0.03 \%$ benzimidazole. To prepare a single-cell isolate from the disease population, a single conidium of each isolate was isolated from the diseased leaves and transferred to the abovementioned surface-disinfested leaflets. All inoculated leaflets in Petri dishes were sealed with parafilm and incubated in a chamber for 10 days with an air temperature of $20 \pm 2^{\circ} \mathrm{C}$ and $16 \mathrm{~h}$ of daily light supplied by warm white fluorescent lamps. The level of irradiance at the top of the Petri dish lid was $50 \mu \mathrm{mol} \mathrm{m} \mathrm{m}^{-2} \mathrm{~s}^{-1}$. This process was repeated every 10th day (with visible powdery mildew) for each single-cell isolate in order to multiply them. After a few cycles of repetition of the above process, leaflets with 10-day-old colonies with proficient sporulation were used to prepare conidial suspension using sterilized distilled water containing Tween-20 (20 $\mu \mathrm{l} / \mathrm{liter})$. The concentration of the suspension was adjusted to $4 \times 10^{4}$ conidia $/ \mathrm{ml}$. Disease-free Espero tomato plants with five fully developed leaves, propagated in a controlled-environment chamber as described above, were moved to five controlled-environment chambers with similar environmental conditions. The tomato plants were then inoculated by spraying each plant with $20 \mathrm{ml}$ of the conidial suspension as described above, with each suspension prepared with dedicated $P$. neolycopersici isolates. For Petri dish level conidia germination experiments, 8- to 9-day-old 
inoculum was used. For conidia germination experiments, $5-\mathrm{cm}$ Petri dishes were used.

\section{UV tolerance and germination recovery of $P$. neolycopersici isolates}

To determine the UV tolerance of the conidia germination process among the collected isolates, Petri-dish-level experiments were performed in controlled-environment chambers with an air temperature of $20 \pm 2{ }^{\circ} \mathrm{C}$ and $\mathrm{RH}$ of $75 \pm 5 \%$. Factorial experiments with four factors (isolates, treatment condition, duration of exposure, and incubation condition) were designed. Conidia were dusted on the surface of a $1 \%$ water agar media contained in Petri dishes. Immediately after inoculation, Petri dishes without lids were exposed to (i) complete darkness or (ii) UV-C with an irradiance of $2 \pm 0.2 \mu \mathrm{mol} \mathrm{m} \mathrm{m}^{-2}$ $\mathrm{s}^{-1}$, or (iii) UV-C with an irradiance of $4 \pm 0.2 \mu \mathrm{mol} \mathrm{m} \mathrm{m}^{-2}$ $\mathrm{s}^{-1}$. The durations of exposure were (i) $10 \mathrm{~s}$, (ii) $20 \mathrm{~s}$, or (iii) 30 s. Immediately after UV treatments, the Petri dishes were sealed and transferred to incubation chambers with lighting conditions of (i) complete darkness for $24 \mathrm{~h}$ or (ii) blue light with an irradiance of $25 \pm 5 \mu \mathrm{mol} \mathrm{m} \mathrm{m}^{-2} \mathrm{~s}^{-1}$ for $2 \mathrm{~h}$ followed by $22 \mathrm{~h}$ of darkness. The conidia were assessed for germination with a light microscope $24 \mathrm{~h}$ after inoculation, and 50 conidia were counted for germination using a handheld tally counter. The percent germination was calculated relative to the non-UV control samples. Conidia with germ tubes equal to or longer than the conidial width were counted as successfully germinated while conidia with germ tubes less than the conidial width were considered nongerminated (Suthaparan et al. 2018). The experiment was repeated twice, with three replicated Petri dishes in each experiment.

UV irradiance with a wavelength peak at $254 \mathrm{~nm}$ was supplied by $120-\mathrm{W}$ germicidal UV-C fluorescent tubes (Light Tech, U.S.A.). Blue light with a peak at a $454 \mathrm{~nm}$ wavelength was provided by a $15-\mathrm{W}$ GreenPower LED module HF blue (Philips, The Netherlands). An Optronic model 756 spectroradiometer (Optronic Laboratories, Orlando, FL, U.S.A.) was used to measure spectral composition and level of irradiances (Supplementary Fig. S1). The temperature and RH of the controlled-environment chambers at Petri dish level were recorded at 5-min intervals using a Priva greenhouse computer (Priva, Zijlweg, The Netherlands) connected to dry and wet bulb thermosensors.

The data analysis was performed using the general linear mixed-effect model, with experimental repeat and replicate as a random effect and isolate type, $\mathrm{UV}$, incubation treatment, and their interactions as fixed effects. Treatment means were separated by way of Tukey's pairwise comparison at $P=0.05$ (Minitab 18.1; Minitab Corp., State College, PA, U.S.A.). Graphs were plotted using SigmaPlot 10.0 (Systat Software, Inc.).

\section{Comparison of sequence similarity of CPF genes among $P$. neolycopersici isolates}

Fungal conidia were harvested from 2-week-old inoculum from diseased tomato plants grown in controlled-environment chambers. Diseased leaves were touched on water agar, and the water agar surfaces were then scraped with a sterile glass slide and conidia were collected in 2-ml Eppendorf tubes. The collected conidia were flash frozen in liquid nitrogen. DNA was extracted using the modified cetyltrimethylammonium bromide method described previously (Robinson et al. 2002), with slight modifications (Supplementary Data S1.1). The quantity and quality of the DNA were measured and checked by a NanoDrop
2000 spectrophotometer (Thermo Fisher Scientific) and agarose gel electrophoresis (Bio-Rad Laboratories), respectively.

The presence of three CPF-like genes and respective coding sequences were identified and characterized with draft genome assembly and transcriptome data of $P$. neolycopersici (isolate As). OINE01015670_T110144, OINE01000912_T103440, and OINE01005061_T102555 were identified in P. neolycopersici (NCBI GenBank accession numbers MT277359, MT277360, and MT277361, respectively), hereafter referred to as $P N 5670$, $P N 0912$, and PN5061, respectively. Known sequences of these genes were divided into 7 to 10 segments with amplicon sizes of 1,006 to $1,135 \mathrm{bp}$ and overlaps of approximately 180 to 200 bp. Primer pairs for each segment were designed (Supplementary Table S1) and three target genes (PN5670, PN0912, and PN5061) were PCR amplified using genomic DNA extracted from five isolates (As, Vf, $\mathrm{Fn}, \mathrm{Sn}$, and $\mathrm{Nl}$ ) as templates.

The target fragments were PCR amplified using a Phusion Green Hot Start II High-Fidelity PCR Master Mix (Thermo Scientific) with the following PCR program: initial denaturation at $98^{\circ} \mathrm{C}$ for $30 \mathrm{~s} ; 35$ cycles of denaturation at $98^{\circ} \mathrm{C}$ for 10 $\mathrm{s}$, annealing at $52^{\circ} \mathrm{C}$ for $30 \mathrm{~s}$, and extension at $72^{\circ} \mathrm{C}$ for $80 \mathrm{~s}$; followed by a final extension at $72^{\circ} \mathrm{C}$ for $10 \mathrm{~min}$. PCR amplicons were analyzed using agarose gel $(0.8 \%)$ electrophoresis (Bio-Rad Laboratories). Amplified fragments were purified using a QIAquick PCR purification kit (Qiagen, Germany). Amplicons were sequenced in both directions (forward and reverse) using the same primers as used in the abovementioned PCR amplification (Supplementary Table S1). Sequencing runs were performed by GATC Biotech, Germany, using an ABI PRISM BigDye Terminator v3.1 Cycle Sequencing Kit on an ABI 3730XL DNA Analyzer (Applied Biosystems).

The raw sequencing reads were trimmed manually using BioEdit v 7.2.5 (Hall 1999) at both ends prior to alignment, then aligned against their respective reference gene sequences (obtained from a draft genome of the As isolate), which were used to design primers. Multiple sequence alignment was carried out by means of MultAlin Interface (Corpet 1988) using default settings (gap penalty at opening and extension) without additional settings. The progressive pairwise alignment of closely related groups of sequences was carried out to achieve a multiple sequence alignment (Corpet 1988). Technical sequencing errors were corrected, and aligned reads were analyzed for the presence of any variations (single-nucleotide variations, insertions, and deletions).

\section{Phylogenetic analysis of CPF-like genes}

Amino acid sequences of CPF-like genes from 10 ascomycetes and 17 other representative members (Table 1) (Supplementary Data S1.2) were searched for and obtained from the NCBI (https://www.ncbi.nlm.nih.gov/), in addition to the deduced amino acid sequences of three CPF-like genes from five isolates of $P$. neolycopersici. Because the amino acid sequences of all five isolates were identical, only three CPF-like genes from one isolate were included in the construction of a phylogenetic tree. These selected CPF-like genes are already reported and extensively studied (Kavakli et al. 2017; Ozturk 2017; Tagua et al. 2015; Thompson and Sancar 2002). A multiple sequence alignment of amino acid sequences was carried out using ClustalW with default parameters. A MEGAX (v.10.0.5) software package was used to perform a phylogenetic analysis and an unrooted tree of sequence data was constructed by means of a neighbor-joining algorithm with 1,000 bootstrap replicates (Tamura et al. 2013). 
TABLE 1

Amino acid sequence GenBank accession numbers of cryptochrome/photolyase family-like genes used in the phylogenetic analysis ${ }^{\mathrm{a}}$

\begin{tabular}{|c|c|c|c|}
\hline Seq. ${ }^{b}$ & Species and taxa & GenBank protein accession number & Designated name \\
\hline 1 & Escherichia coli & NP_415236.1 & E. coli DNA PHR \\
\hline 2 & Bacillus cereus & WP_001179969.1 & B. cereus PHR like \\
\hline 3 & Bacillus thuringiensis & WP_001179974.1 & B. thuringiensis PHR like \\
\hline 4 & Caulobacter vibrioides & YP_002516868.1 & C. vibrioides PHR like \\
\hline 5 & Methanobacter thermoautotrophicus & WP_010876537.1 & M. thermoautotrophicus DNA PHR \\
\hline 6 & Salmonella typhimurium & NP_459694.1 & S. enterica DNA PHR like \\
\hline 7 & Vibrio cholerae & NP_231448.1 & V. cholerae DNA PHR like \\
\hline 8 & Agrobacterium tumefaciens & WP_038490669.1 & A. tumefaciens DNA PHR like \\
\hline 9 & Anacystis nidulans & WP_011377448.1 & A. nidulans DNA PHR \\
\hline 10 & Saccharomyces cerevisiae & NP_015031.1 & S. cerevisiae PHR1 \\
\hline 11 & Alternaria alternata & XP_018380931.1 & A. alternata cryptochrome DASH like \\
\hline 12 & Alternaria alternata & XP_018388382.1 & A. alternata cryptochrome 2 \\
\hline 13 & Aspergillus nidulans & XP_657991.1 & A. nidulans PHR like \\
\hline 14 & Aspergillus niger & XP_001397458.2 & A. nidulans DNA PHR \\
\hline 15 & Aspergillus niger & XP_001402217.1 & A. nidulans DNA PHR like \\
\hline 16 & Blumeria graminis f. sp. hordei & CCU75260.1 & B. graminis f. sp. hordei cryptochrome DASH \\
\hline 17 & Blumeria graminis f. sp. hordei & CCU75653.1 & B. graminis f. sp. hordei cryptochrome like \\
\hline 18 & Blumeria graminis f. sp. hordei & CCU77936.1 & B. graminis f. sp. hordei DNA PHR \\
\hline 19 & Blumeria graminis f. sp. tritici & EPQ63205.1 & B. graminis f. sp. tritici DNA PHR \\
\hline 20 & Blumeria graminis f. sp. tritici & EPQ63708.1 & B. graminis f. sp. tritici DNA PHR \\
\hline 21 & Blumeria graminis f. sp. tritici & EPQ64280.1 & B. graminis f. sp. tritici DNA PHR \\
\hline 22 & Botrytis cinerea & XP_001548442.2 & B. cinerea Bccry 1 \\
\hline 23 & Botrytis cinerea & XP_024550731.1 & B. cinerea Bccry 2 \\
\hline 24 & Erysiphe necator & KHJ30144.1 & E. necator DNA PHR like \\
\hline 25 & Erysiphe necator & KHJ34441.1 & E. necator DNA PHR like \\
\hline 26 & Erysiphe necator & KHJ35948.1 & E. necator cryptochrome DASH like \\
\hline 27 & Fusarium fujikuroi & XP_023425040.1 & F. fujikuroi PHR like \\
\hline 28 & Fusarium fujikuroi & XP_023428189.1 & F. fujikuroi DNA PHR like \\
\hline 29 & Fusarium fujikuroi & XP_023431896.1 & F. fujikuroi DNA PHR like \\
\hline 30 & Fusarium oxysporum & XP_018235323.1 & F. oxysporum f. sp. lycopersici cryptochrome \\
\hline 31 & Golovinomyces cichoracearum & RKF54075.1 & G. cichoracearum DNA PHR \\
\hline 32 & Golovinomyces cichoracearum & RKF65157.1 & G. cichoracearum cryptochrome- 2 \\
\hline 33 & Golovinomyces cichoracearum & RKF80931.1 & G. cichoracearum cryptochrome DASH like \\
\hline 34 & Neurospora crassa & XP_964834.2 & N. crassa DNA PHR \\
\hline 35 & Neurospora crassa & XP_965722.3 & N. crassa cryptochrome DASH like \\
\hline 36 & Pseudoidium neolycopersici & QKE45385.1 & P. neolycopersici cryptochrome like* \\
\hline 37 & Pseudoidium neolycopersici & QKE45387.1 & P. neolycopersici DNA PHR* \\
\hline 38 & Pseudoidium neolycopersici & QKE45389.1 & P. neolycopersici cryptochrome DASH like* \\
\hline 39 & Trichoderma atroviride & XP_013938084.1 & T. atroviride DNA PHR 1 \\
\hline 40 & Trichoderma atroviride & XP_013941790.1 & T. atroviride PHR like \\
\hline 41 & Trichoderma atroviride & XP_013942035.1 & T. atroviride DNA PHR like \\
\hline 42 & Arabidopsis thaliana & NP_566520.1 & A. thaliana DNA PHR family protein \\
\hline 43 & Arabidopsis thaliana & NP_568461.3 & A. thaliana cryptochrome-3 \\
\hline 44 & Arabidopsis thaliana & NP_171935.1 & A. thaliana cryptochrome- 2 \\
\hline 45 & Arabidopsis thaliana & NP_563906.1 & A. thaliana DNA PHR II \\
\hline 46 & Arabidopsis thaliana & NP_567341.1 & A. thaliana cryptochrome-1 \\
\hline 47 & Brassica rapa & NP_001288891.1 & B. rapa cryptochrome-1 \\
\hline 48 & Brassica rapa & XP_009110652.1 & B. rapa DNA PHR \\
\hline 49 & Brassica rapa & XP_009111106.1 & B. rapa cryptochrome- 2 \\
\hline 50 & Brassica rapa & XP_009111643.1 & B. rapa cryptochrome DASH like \\
\hline 51 & Brassica rapa & XP_009115387.1 & B. rapa (6-4) PHR \\
\hline 52 & Glycine max & NP_001235220.1 & G. $\max$ cryptochrome- 2 \\
\hline 53 & Glycine $\max$ & NP_001238710.2 & G. $\max$ CPD PHR \\
\hline 54 & Glycine $\max$ & NP_001242152.1 & G. $\max$ cryptochrome-1 like \\
\hline 55 & Zea mays & NP_001130580.1 & Z. mays type II CPD DNA PHR \\
\hline 56 & Zea mays & XP_008677763.1 & Z. mays cryptochrome- 2 \\
\hline 57 & Drosophila melanogaster & NP_724274.1 & D. melanogaster (6-4) PHR \\
\hline 58 & Drosophila melanogaster & NP_523653.2 & D. melanogaster \\
\hline 59 & Drosophila melanogaster & NP_732407.1 & D. melanogaster cryptochrome \\
\hline 60 & Homo sapiens & XP_024304612.1 & H. sapiens cryptochrome-1 \\
\hline 61 & Homo sapiens & NP_066940.3 & H. sapiens cryptochrome- 2 \\
\hline 62 & Gallus gallus & NP_989575.1 & G. gallus cryptochrome-2 \\
\hline 63 & Gallus gallus & NP_989576.1 & G. gallus cryptochrome-1 \\
\hline 64 & Mus musculus & NP_031797.1 & M. musculus cryptochrome-1 \\
\hline 65 & Mus musculus & NP_034093.1 & M. musculus cryptochrome- 2 \\
\hline
\end{tabular}

a The highlighted (bold) sequences of representative model organisms, including three cryptochrome/photolyase family-like genes (indicated by an asterisk [*]) from Pseudoidium neolycopersici, were also used in amino acid alignment analysis. PHR $=$ photolyase; DASH $=$ Drosophila, Arabidopsis, Synechocystis, Human; and CPD = cyclobutane pyrimidine dimer.

Sequence number. 


\section{Amino acid sequence alignment and analysis}

The deduced amino acid sequence for an open reading frame (ORF) of three CPF-like genes (PN5670, PN0912, and $P N 5061)$ of $P$. neolycopersici was compared with amino acid sequences of nine other representative members of CPF (Supplementary Data S1.2). The selected members are extensively studied model organisms. These CPF sequences were also retrieved from NCBI (https://www.ncbi.nlm.nih.gov/) and also included in the phylogenetic analysis. Alignment was performed using Vector NTI Advance 11.5.4 (Invitrogen).

\section{RESULTS}

\section{UV tolerance and germination recovery of $P$. neolycopersici isolates}

All factors of isolate type, level of UV irradiance, duration of UV exposure, incubation light condition, and isolate-incubation light interaction showed a significant effect on the germination efficiency of conidia $(P<0.0001)$. Independent of isolate type, UV treatments of 2 or $4 \mu \mathrm{mol} \mathrm{m}{ }^{-2} \mathrm{~s}^{-1}$ followed by dark incubation significantly reduced conidia germination $(<30 \%)$ compared with the non-UV control (>90\%) (Fig. 1A and C). Germination efficiency was found to decrease with increased duration of UV exposure.

The conidial germination efficiency of all isolates was recovered as in the non-UV control, when samples were incubated with blue light after brief UV treatment. The highest germination efficiency was recorded in the isolate from the Netherlands ( 78 to $89 \%$ ) followed by the isolate from Finnøy (62 to $77 \%$ ). All other isolates showed slightly lower germination efficiency of conidia (35 to 66\%), independent of UV irradiance level and duration of exposure (Fig. 1B and D).

\section{Nucleotide sequence analysis of CPF genes in P. neolycopersici isolates}

A nucleotide sequence analysis of three CPF genes (PN5670, $P N 0912$, and PN5061) showed no polymorphism among the five $P$. neolycopersici isolates collected from different geographical

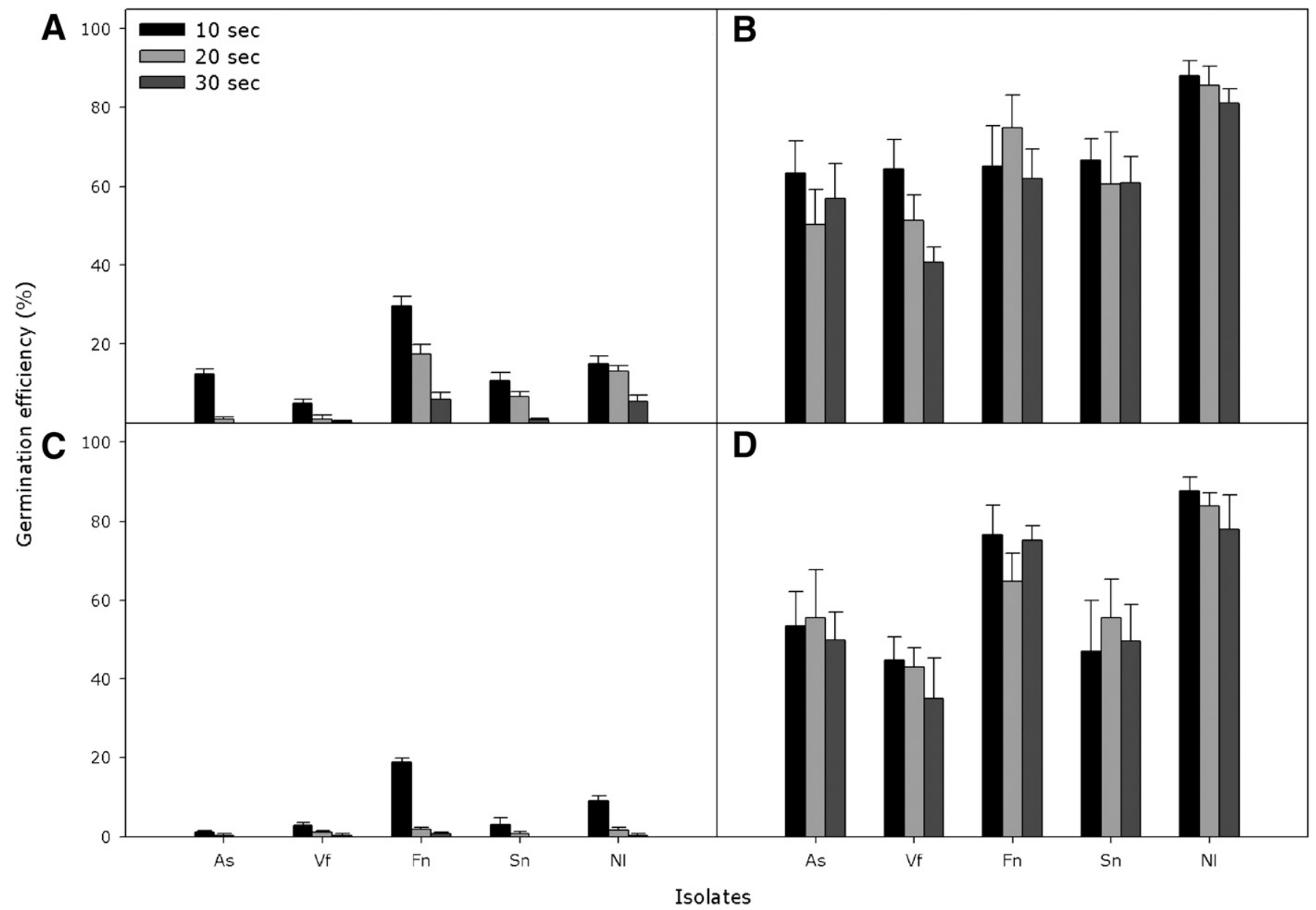

FIGURE 1

Effect of brief UV treatments and subsequent incubation wavelengths on the conidial germination efficiency of five different isolates of Pseudoidium neolycopersici. Conidia from 8- to 9-day-old colonies with profound sporulation collected from isolates from Akershus (As), Vestfold (Vf), Finnøy (Fn), Sandnes (Sn), or the Netherlands (NI) were dusted on a water agar surface in 5-cm Petri dishes, and exposed to brief UV-C (peak of $254 \mathrm{~nm}$ ) treatments $\left(10,20\right.$, or $30 \mathrm{~s}$ ) of $\mathbf{A}, 2 \pm 0.2 \mu \mathrm{mol} \mathrm{m}{ }^{-2} \mathrm{~s}^{-1}$ followed by complete darkness; $\mathbf{B}, 2 \pm 0.2 \mu \mathrm{mol}$ $\mathrm{m}^{-2} \mathrm{~s}^{-1}$ followed by $2 \mathrm{~h}$ of blue light $\left(454 \mathrm{~nm}\right.$ ) followed by complete darkness; C, $4 \pm 0.2 \mu \mathrm{mol} \mathrm{m} \mathrm{m}^{-2} \mathrm{~s}^{-1}$ followed by complete darkness; or $\mathrm{D}, 4 \pm 0.2 \mu \mathrm{mol} \mathrm{m}{ }^{-2} \mathrm{~s}^{-1}$ followed by $2 \mathrm{~h}$ of blue light $(454 \mathrm{~nm}$ ) followed by complete darkness. Samples were assessed for conidia germination $24 \mathrm{~h}$ after inoculation under a light microscope. The percent germination was calculated relative to non-UV-treated control samples. Values are the mean \pm standard error of two experiments, each with three replicates $(n=6)$. 
regions. The alignments of PCR-amplified and sequenced products for above three genes are presented in Supplementary Figure S2.A, B, and C.

\section{Phylogenetic analysis}

Phylogenetic analysis showed that three CPF-like genes (PN5670, PN0912, and PN5061) from P. neolycopersici clustered with CRY, type I cyclobutane pyrimidine dimer (CPD) PHR, and CRY-Drosophila, Arabidopsis, Synechocystis, Human (DASH), respectively, together with the other CPFlike genes (Fig. 2).

\section{Amino acid sequence analysis of P. neolycopersici CPF-like genes}

Amino acid sequences of the ORF for three CPF-like genes identified in $P$. neolycopersici were compared with the ORF of the other representative members of CPF-like proteins, CRY, (6-4) PHR, CPD PHR, and CRY-DASH, which belong to humans, plants, bacteria, fungi, and insects. PN5670 showed a 34 to $38 \%$ sequence homology to human CRY and (6-4) PHR from Drosophila melanogaster and Arabidopsis thaliana. PN5061 showed a $48 \%$ sequence homology to CRY-DASH from Neurospora crassa and 32\% homology to CRY-DASH from A. thaliana and cry1 from Vibrio cholera. PN0912 showed a 57\% sequence homology to CPD PHR from N. crassa and 35 to $41 \%$ homology to E. coli and Anacystis nidulans (Fig. 3).

In addition, PN0912 showed a protruding N-terminal end of 134 amino acid residues similar to $N$. crassa PHR, which was 106 amino acid residues long, and also showed sequence homology in this region. These protruding amino $(\mathrm{N})$ terminal ends were not observed in E. coli and A. nidulans PHR (Fig. 3).

Amino acid sequence alignment also showed three crucial residues (W277, M345, and N378) as well as a tryptophan triad (W306, W359, and W382), which are highly conserved in CPF members. The alignment showed higher conservation of amino acid residues in the C-terminal ends compared with $\mathrm{N}$-terminal ends. It was also observed that, among all $\mathrm{CPF}$ protein members, CRY have considerably longer C-terminal ends compared with PHR, which were observed in $P N 5670$ and PN5061 (Figs. 3 and 4).

\section{DISCUSSION}

The variation in germination recovery among the tested isolates of $P$. neolycopersici indicates that there is a difference in the efficiency of PHR-mediated UV damage recovery. However, the identical sequences of PHR genes among all tested $P$. neolycopersici isolates (Supplementary Fig. S2) suggests that this biological variation in germination recovery is not due to the sequence variation in the coding regions of PHR genes. PHR are globular proteins having one catalytic cofactor of FAD and an additional cofactor that serves as photoantenna (Vechtomova et al. 2020). Depending on the type and number of additional photoantenna present, the absorption and action spectrum will vary. This can lead to variation in the efficiency of PHR-mediated UV damage recovery. In our previous study, we identified FAD and MTHF as possible cofactors present in $P$. neolycopersici PHR. However, this was based on a structure prediction model and optical properties of heterologous PHR protein expressed in E. coli (Pathak et al. 2020). Despite the potential variation in cofactors among the isolates of the same species, an examination of optical properties of native proteins of $P$. neolycopersici PHR may explain the possible reason for underlying biological variation. In addition to optical properties of PHR, the expression level of a PHR gene and its translation efficiency may also affect the efficiency of PHR-mediated UV damage recovery. Detailed studies on the promoter architecture and transcription binding sites may have revealed information beyond the findings of this study. In filamentous fungi, it has been demonstrated that PHR gene upregulation by blue/visible light requires a blue-light-responsive cis element in the promotor region of the gene (Kihara et al. 2004). However, little is known about the role and variation of the cis element among isolates of the same species. It has been reported that the expression of PHR was induced by white collar 1 in Ustilago maydis (Brych et al. 2016). Hence, it is also important to examine the role of regulatory genes involved in the expression of PHR.

The same UV dose showed different effect patterns of UV on the germination of isolates. Two isolates seemed more tolerant, whereas the remaining three isolates were sensitive to the same dosage of UV (Fig. 1). This variation in UV response of fungal isolates may reflect the natural adaptations to their different environmental conditions. The daily optical environment in which organisms thrive, and how they adapt or optimize their genomic and proteomic features to survive in that particular environment, is an important aspect (Zhou et al. 2007). UV radiation has shown great potential in controlling powdery mildew diseases in different crops at the greenhouse level (Suthaparan et al. 2012a, b, 2014, 2016a). Exploring the tolerance or sensitivity to UV of fungal isolates is considered to be an important step in identifying a promising UV dose in UVmediated powdery mildew disease management. The variation in response to UV among isolates may also be explained by one of the important genomic features, base composition; most importantly, the dinucleotide composition in the genome of a species (Zhou et al. 2007). A major type of UV-induced lesion is CPDs, which are formed between two adjacent pyrimidine nucleotides (Sancar 2008). Therefore, a high pyrimidine content in a genome may lead to the formation of more CPDs that, in turn, leads to a lower repair efficiency or a higher cell death.

All CPF members possess a core domain approximately 500 amino acid residues long but differ significantly in relation to $\mathrm{N}$ - and C-terminal extensions (Chaves et al. 2006). The C-terminal ends are highly conserved compared with $\mathrm{N}$-terminal ends as a catalytic cofactor (FAD), which is common to all CPF members, binds in the C-terminal ends (Essen and Klar 2006). The protruding amino terminal ends which were observed in $P$. neolycopersici and $N$. crassa PHR showed a significant similarity in amino acid residues (Figs. 3 and 4). These amino acid residues have homology to a known cleavage site that enables the transport of the mitochondrial leucyl-tRNA synthetase into mitochondria in $N$. crassa (Chow et al. 1989). Saccharomyces cerevisiae also possess protruding amino terminal ends which play an important role in transporting PHR into mitochondria, and are capable of repairing pyrimidine dimers in nuclei as well as in mitochondria (Yasui et al. 1992). On the other hand, S. cerevisiae expressing an $E$. coli $\mathrm{PHR}$ was able to repair a fraction of pyrimidine dimers present in the nucleus but not in mitochondria, likely because E. coli PHR lacks a protruding N-terminal signal sequence, which is required for subcellular localization to these organelles (Prakash 1975; Yasui et al. 1992). These protruding amino terminal ends are characteristics only found in fungal PHR, which suggests that they may act as signal sequences with an 


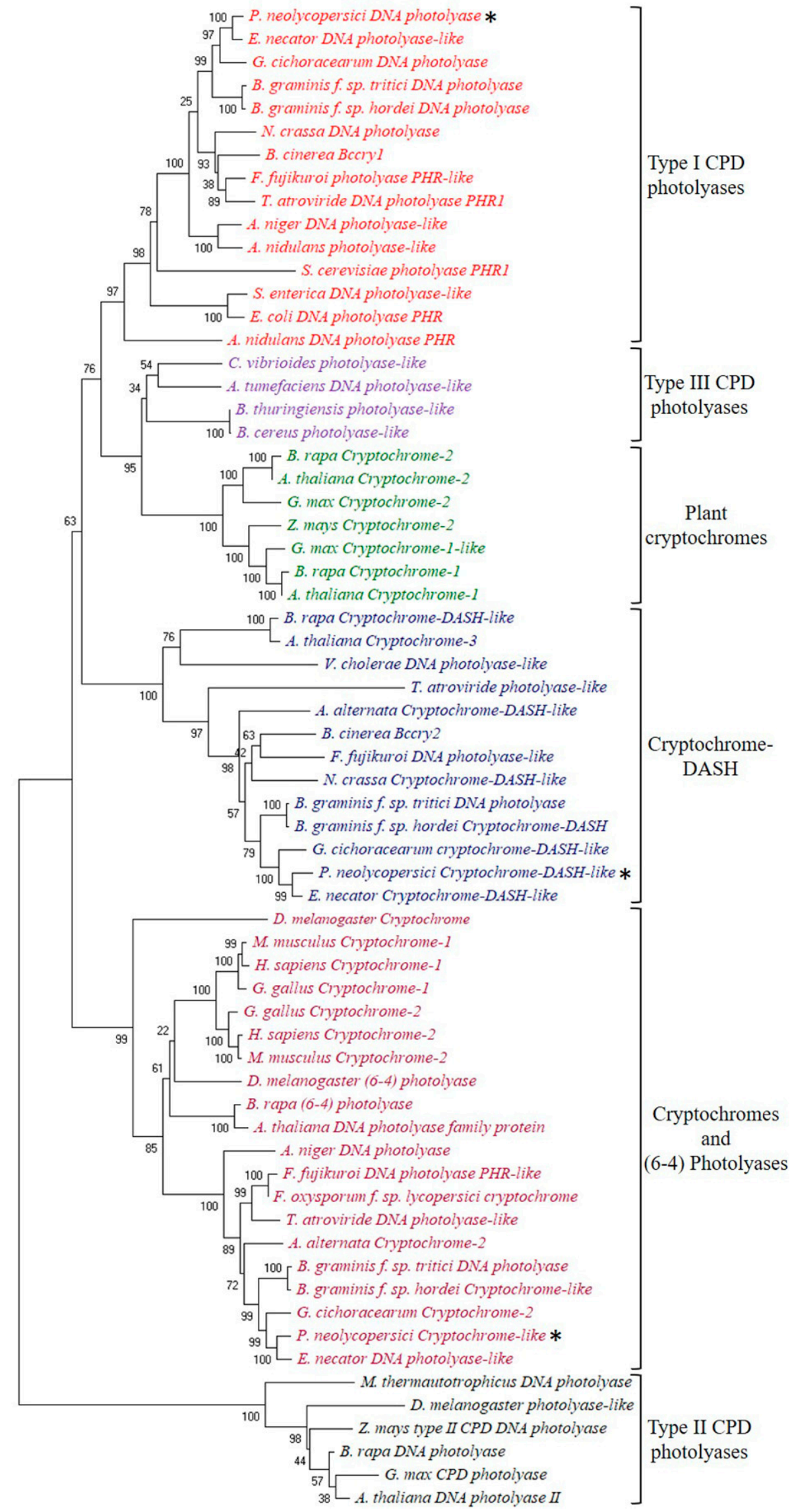

FIGURE 2

Phylogenetic analysis of cryptochrome/photolyase family (CPF)-like genes, including CPF-like genes from Pseudoidium neolycopersici (indicated by an asterisk $\left.{ }^{*}\right]$ ). The neighbor- joining method was used to construct an unrooted phylogenetic tree. The bootstrap probability of 1,000 replicates is expressed in percentages. 


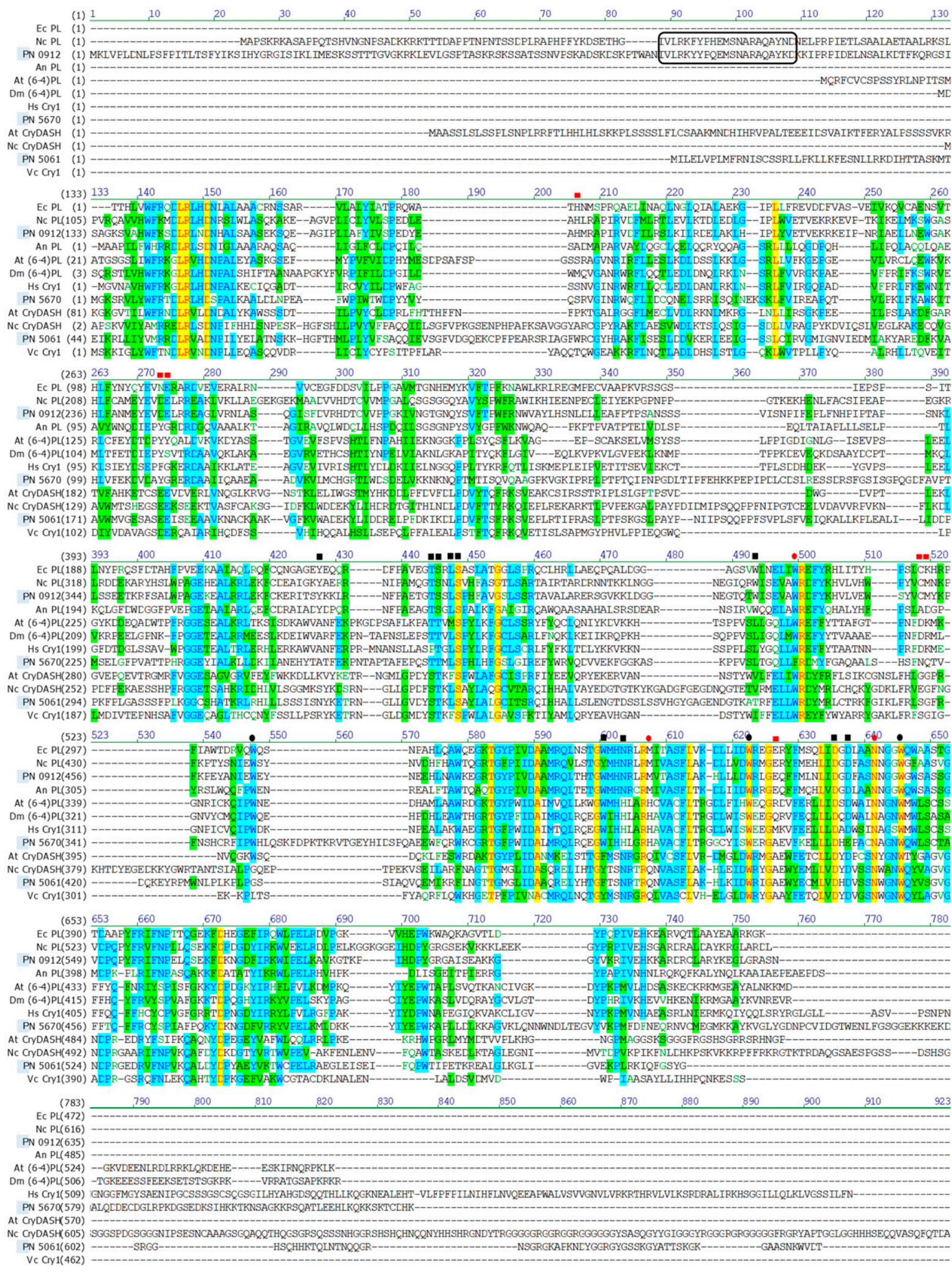

FIGURE 3

Comparative analysis of amino acid sequences of three cryptochrome/photolyase family (CPF)-like genes of Pseudoidium neolycopersici (PN5670, PN0912, and PN5061) with other CPF members from Anacystis nidulans (An); Escherichia coli (Ec); Neurospora crassa (Nc); Arabidopsis thaliana (At); Drosophila melanogaster (Dm); Homo sapiens (Hs); and Vibrio cholerae (Vc). Alignment was performed using the Vector NTI software program. The black rectangular box showing the homology region, which includes an identical probable signal sequence, RKYYPQ in P. neolycopersici to RKFYPH in N. crassa. Colors: yellow = identical; green = block conservation; and blue $=$ highly conserved. Symbols: black squares = flavin adenine dinucleotide interactions; red squares $=5,10$-methenyltetrahydrofolate interactions; black circles = tryptophan triad (W306, W359, and W382); and red circles = crucial residues (W277, M345, and N378). Abbreviations: $\mathrm{PL}=$ cyclobutane pyrimidine dimer photolyase; $(6-4) \mathrm{PL}=(6-4)$ photolyase; Cry = cryptochrome; and cry-DASH = cryptochrome-Drosophila, Arabidopsis, Synechocystis, Human. 


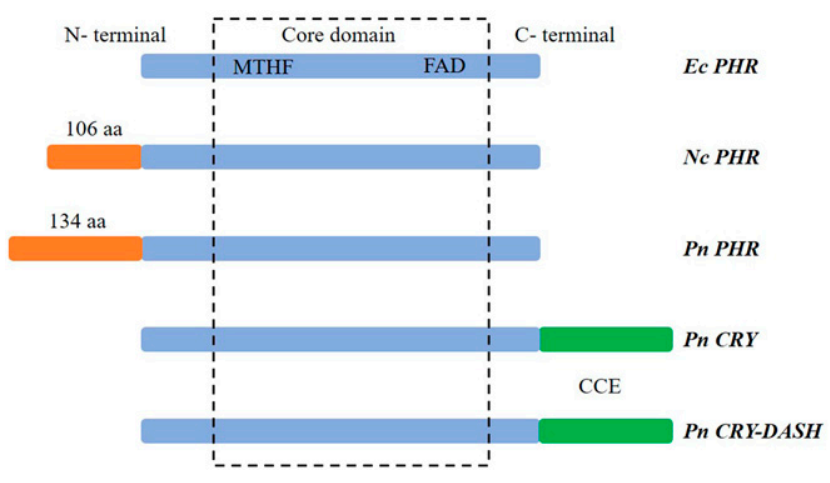

FIGURE 4

Diagrammatic representation of the comparison of cryptochrome (CRY)/photolyase family (CPF)-like genes (PN5670, PN0912, and PN5061) identified in Pseudoidium neolycopersici (Pn) with Escherichia coli (Ec) and Neurospora crassa (Nc) photolyase, showing an $\mathrm{N}$-terminal end, core domain consisting of a flavin adenine dinucleotide (FAD) and 5,10-methenyltetrahydrofolate (MTHF), and a C-terminal end. Fungal photolyases (phr) of $N$. crassa and $P$. neolycopersici showed protruding $\mathrm{N}$-terminal ends of 106 and 134 amino acid residues long, respectively. P. neolycopersici CRY and CRY-Drosophila, Arabidopsis, Synechocystis, Human (CRY-DASH) show Cry C-terminal extensions (CCE).

important role in PHR translocation in $P$. neolycopersici (Fig. 3). Additionally, a tryptophan triad (trp triad) was found to be conserved among PHR, including class I, class II CPD PHR, and (6-4) PHR. Previously, it was reported that this trp triad provides an alternative electron-transfer pathway (W306 $\rightarrow \mathrm{W} 359 \rightarrow$ W382 $\rightarrow$ FADH $^{\bullet}$ ) (Fig. 3) (Weber 2005; Zhang et al. 2017). Another three critical residues (W277, M345, and N378) (Fig. 3) are found to be distributed in this superfamily. Residue trp277 (W277) is crucial for substrate binding and plays a role in CPD splitting. Met345 (M345) is restricted to CPD PHR because it discriminates CPD PHR from rest of the superfamily. Asn378 (N378) is highly conserved in the whole superfamily because it stabilizes the neutral FAD radical (Xu and Zhu 2010).

Among different species, CPF members share a highly conserved core domain, which is responsible for their function. It is well acknowledged that the DNA sequences of a gene are highly conserved within the same species and even in distantly related species (Reaume and Sokolowski 2011). Therefore, the fact that the results showed highly conserved nucleotide sequences of all three CPF members of $P$. neolycopersici in all five isolates collected from different regions is justifiable. Nonetheless, the functions of protruding N-terminal ends in PHR and Cry C-terminal extensions in CRY require further investigation in relation to powdery mildews.

\section{ACKNOWLEDGMENTS}

We thank Prof. Hans Ragnar Gislerød (collection of pathogen isolates) and K. Svinnset for help in the maintenance of isolates, and other technical staff at the Centre for Controlled Environment Plant Research, Norwegian University of Life Sciences, for their excellent support and assistance.

\section{LITERATURE CITED}

Brych, A., Mascarenhas, J., Jaeger, E., Charkiewicz, E., Pokorny, R., Bölker, M., Doehlemann, G., and Batschauer, A. 2016. White collar 1-induced photolyase expression contributes to UV-tolerance of Ustilago maydis. MicrobiologyOpen 5:224-243.

Chaves, I., Yagita, K., Barnhoorn, S., Okamura, H., van der Horst, G. T. J., and Tamanini, F. 2006. Functional evolution of the photolyase/ cryptochrome protein family: Importance of the $\mathrm{C}$ terminus of mammalian CRY1 for circadian core oscillator performance. Mol. Cell. Biol. 26:1743-1753.

Chow, C. M., Metzenberg, R. L., and Rajbhandary, U. L. 1989. Nuclear gene for mitochondrial leucyl-tRNA synthetase of Neurospora crassa: Isolation, sequence, chromosomal mapping, and evidence that the leu-5 locus specifies structural information. Mol. Cell. Biol. 9:4631-4644.

Corpet, F. 1988. Multiple sequence alignment with hierarchical clustering. Nucleic Acids Res. 16:10881-10890.

Essen, L. O., and Klar, T. 2006. Light-driven DNA repair by photolyases. Cell. Mol. Life Sci. 63:1266-1277.

Hall, T. A. 1999. BioEdit: A user-friendly biological sequence alignment editor and analysis program for windows 95/98/NT. Nucleic Acids Symp. Ser. 41:95-98.

Jans, J., Schul, W., Sert, Y. G., Rijksen, Y., Rebel, H., Eker, A. P. M., Nakajima, S., van Steeg, H., de Gruijl, F. R., Yasui, A., Hoeijmakers, J. H. J., and van der Horst, G. T. J. 2005. Powerful skin cancer protection by a CPD- photolyase transgene. Curr. Biol. 15:105-115.

Jenkins, G. J. S., Burlinson, B., and Parry, J. M. 2000. The polymerase inhibition assay: A methodology for the identification of DNAdamaging agents. Mol. Carcinog. 27:289-297.

Jones, H., Whipps, J. M., and Gurr, S. J. 2001. The tomato powdery mildew fungus Oidium neolycopersici. Mol. Plant Pathol. 2:303-309.

Kavakli, I. H., Baris, I., Tardu, M., Gül, Ş., Öner, H., Çal, S., Bulut, S., Yarparvar, D., Berkel, C.., Ustaoğlu, P., and Aydın, C. 2017. The photolyase/cryptochrome family of proteins as DNA repair enzymes and transcriptional repressors. Photochem. Photobiol. 93:93-103.

Kihara, J., Moriwaki, A., Matsuo, N., Arase, S., and Honda, Y. 2004 Cloning, functional characterization, and near-ultraviolet radiationenhanced expression of a photolyase gene (PHR1) from the phytopathogenic fungus Bipolaris oryzae. Curr. Genet. 46:37-46.

Lebeda, A., Mieslerová, B., Petřivalský, M., Luhová, L., Špundová, M., Sedlářová, M., Nožková-Hlaváčková, V., and Pink, D. A. C. 2017. Review of tomato powdery mildew-A challenging problem for researchers, breeders and growers. Acta Hortic. 1159:107-116.

Manova, V., and Gruszka, D. 2015. DNA damage and repair in plantsFrom models to crops. Front. Plant Sci. 6:885.

Mei, Q., and Dvornyk, V. 2015. Evolutionary history of the photolyase/ cryptochrome superfamily in eukaryotes. PLoS One 10:e0135940.

Ozturk, N. 2017. Phylogenetic and functional classification of the photolyase/cryptochrome family. Photochem. Photobiol. 93:104-111.

Pathak, R., Ergon, A., Stensvand, A., Gisler d, H. R., Solhaug, K. A., Davidson, L. C., and Suthaparan, A. 2020. Functional characterization of Pseudoidium neolycopersici photolyase reveals mechanisms behind the efficacy of nighttime UV on powdery mildew suppression. Front. Microbiol. 11:1091.

Pathak, R., Sundaram, A., Davidson, L. C., Solhaug, K. A., Stensvand, A., Gisler d, H. R., and Suthaparan, A. 2017. Sensing of UV and visible light by powdery mildew pathogens. Phytopathology 107:47-48.

Prakash, L. 1975. Repair of pyrimidine dimers in nuclear and mitochondrial DNA of yeast irradiated with low doses of ultraviolet light. J. Mol. Biol. 98:781-795.

Reaume, C. J., and Sokolowski, M. B. 2011. Conservation of gene function in behaviour. Philos. Trans. R. Soc. Lond. B Biol. Sci. 366:2100-2110.

Robinson, H. L., Ridout, C. J., Sierotzki, H., Gisi, U., and Brown, J. K. M. 2002. Isogamous, hermaphroditic inheritance of mitochondrion-encoded resistance to Qo inhibitor fungicides in Blumeria graminis f. sp. tritici. Fungal Genet. Biol. 36:98-106.

Sancar, A. 1994. Structure and function of DNA photolyase. Biochemistry $33: 2-9$.

Sancar, A. 2008. Structure and function of photolyase and in vivo enzymology: $50^{\text {th }}$ anniversary. J. Biol. Chem. 283:32153-32157.

Sancar, A., Smith, F. W., and Sancar, G. B. 1984. Purification of Escherichia coli DNA photolyase. J. Biol. Chem. 259:6028-6032.

Sinha, R. P., and Häder, D. P. 2002. UV-induced DNA damage and repair: A review. Photochem. Photobiol. Sci. 1:225-236. 
Suthaparan, A., Pathak, R., Solhaug, K. A., and Gisler־ð, H. R. 2018. Wavelength dependent recovery of UV-mediated damage: Tying up the loose ends of optical based powdery mildew management. J. Photochem. Photobiol. B 178:631-640.

Suthaparan, A., Solhaug, K. A., Bjugstad, N., Gisler``d, H. R., Gadoury, D. M., and Stensvand, A. 2016a. Suppression of powdery mildews by UV-B: Application frequency and timing, dose, reflectance, and automation. Plant Dis. 100:1643-1650.

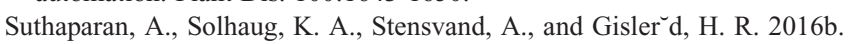
Determination of UV action spectra affecting the infection process of Oidium neolycopersici, the cause of tomato powdery mildew. J. Photochem. Photobiol. B 156:41-49.

Suthaparan, A., Solhaug, K. A., Stensvand, A., and Gisler־ d, H. R. 2017. Daily light integral and day light quality: Potentials and pitfalls of nighttime UV treatments on cucumber powdery mildew. J. Photochem. Photobiol. B 175:141-148.

Suthaparan, A., Stensvand, A., Solhaug, K. A., Torre, S., Mortensen, L. M.,

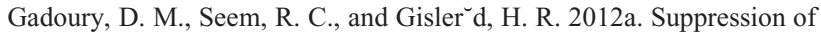
powdery mildew (Podosphaera pannosa) in greenhouse roses by brief exposure to supplemental UV-B radiation. Plant Dis. 96:1653-1660.

Suthaparan, A., Stensvand, A., Solhaug, K. A., Torre, S., Telfer, K. H., Ruud, A. K., Davidson, L. C., Mortensen, L., Gadoury, D. M., Seem,

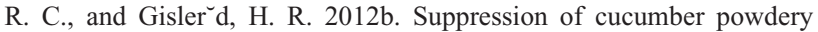
mildew by UV-B is affected by background light quality. (Abstr.) Phytopathology 102:S4.116.

Suthaparan, A., Stensvand, A., Solhaug, K. A., Torre, S., Telfer, K. H., Ruud, A. K., Mortensen, L. M., Gadoury, D. M., Seem, R. C., and

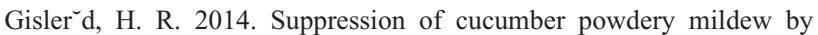
supplemental UV-B radiation in greenhouses can be augmented or reduced by background radiation quality. Plant Dis. 98:1349-1357.

Tagua, V. G., Pausch, M., Eckel, M., Gutiérrez, G., Miralles-Durán, A., Sanz, C., Eslava, A. P., Pokorny, R., Corrochano, L. M., and Batschauer, A. 2015. Fungal cryptochrome with DNA repair activity reveals an early stage in cryptochrome evolution. Proc. Natl. Acad. Sci. U.S.A. 112:15130-15135.

Tamura, K., Stecher, G., Peterson, D., Filipski, A., and Kumar, S. 2013. MEGA6: Molecular evolutionary genetics analysis version 6.0. Mol. Biol. Evol. 30:2725-2729.

Thompson, C. L., and Sancar, A. 2002. Photolyase/cryptochrome bluelight photoreceptors use photon energy to repair DNA and reset the circadian clock. Oncogene 21:9043-9056.

Tornaletti, S., Reines, D., and Hanawalt, P. C. 1999. Structural characterization of RNA polymerase II complexes arrested by a cyclobutane pyrimidine dimer in the transcribed strand of template DNA. J. Biol. Chem. 274:24124-24130.

Vechtomova, Y. L., Telegina, T. A., and Kritsky, M. S. 2020. Evolution of proteins of the DNA photolyase/cryptochrome family. Biochemistry (Moscow) 85:131-153.

Weber, S. 2005. Light-driven enzymatic catalysis of DNA repair: A review of recent biophysical studies on photolyase. Biochim. Biophys. Acta Bioenerg. 1707:1-23.

$\mathrm{Xu}$, L., and Zhu, G. 2010. The roles of several residues of Escherichia coli DNA photolyase in the highly efficient photo-repair of cyclobutane pyrimidine dimers. J. Nucleic Acids 2010:794782.

Yajima, H., Inoue, H., Oikawa, A., and Yasui, A. 1991. Cloning and functional characterization of a eucaryotic DNA photolyase gene from Neurospora crassa. Nucleic Acids Res. 19:5359-5362.

Yasui, A., Yajima, H., Kobayashi, T., Eker, A. P. M., and Oikawa, A. 1992. Mitochondrial DNA repair by photolyase. Mutat. Res. 273:231-236.

Zhang, M., Wang, L., and Zhong, D. 2017. Photolyase: Dynamics and mechanisms of repair of sun-induced DNA damage. Photochem. Photobiol. 93:78-92.

Zhou, P., Wen, J., Oren, A., Chen, M., and Wu, M. 2007. Genomic survey of sequence features for ultraviolet tolerance in Haloarchaea (family Halobacteriaceae). Genomics 90:103-109. 